\title{
Optimization Method of Concurrency Scheduling of Graphical Database Transaction
}

\author{
Nan Lin ${ }^{1, a}$, Li Dongming ${ }^{1, a}$, Han Yongqi ${ }^{1, a}$ \\ ${ }^{1}$ Computer Science Department, Information and Technology College, Jilin Agriculture University, \\ Xincheng Street, 130118, Changchun, China \\ alinnankitty@foxmail.com
}

Keywords: concurrent scheduling; artificial fish swarm algorithm; database transaction

\begin{abstract}
The transaction scheduling problem of graphic database is researched. The reasonable arrangement of the database transaction commit is taken in order, and the fitting first heuristic algorithm and artificial fish swarm algorithm are combined. The graphic database transaction scheduling problem in the massive database is calculated. The foraging and clustering mathematical models are constructed in the artificial fish swarm, the constraint conflict drawbacks aresolved. The optimal scheduling is obtained. The experimental results show that the scheduling problem of hybrid artificial fish swarm algorithm is solved, and the performance of massive database transaction scheduling is improved, the database transaction scheduling is quick and effective. The serializable processing of database transaction is ensured.
\end{abstract}

\section{Introduction}

Currently, the graphics database system is a hot research field, it is one of the core problem how to ensure the consistency of the global execution of concurrent transactions in the database in the process of operation. The necessary conditions for maintaining the consistency of executing global transactions is to ensure that concurrent executing global transactions is serializable in cross site. Therefore, how to ensure that concurrent executing global transactions cross site is important, graphics database concurrency scheduling process is key to ensure the serializability of graphics database scheduling. Mehrotra proposed the commit transaction manager chart centric (GTM) scheduling algorithm, the TM1 and TM2 algorithms are proposed through a diagram to guarantee the global commit serializability. Because GTM cannot detected by indirect conflicts between global transactions and local affairs, the above algorithms have not good performance, it cannot ensure the global stability, it has difficulty in a multi database environment[1-3].

In order to solve the local transaction execution problem, due to control algorithm of GTM cannot detect the global transactions and local affairs, the indirect conflict problem is solved. This paper proposes an efficient scheduling algorithm of hybrid artificial fish swarm algorithm for massive database based on the fitting first heuristic algorithm and artificial fish swarm algorithm. The two algorithms are combined, and the graphic database of things is obtained. The fish foraging, cluster mathematical models are constructed solve constraint conflict, malpractice, and the optimal scheduling of affairs is obtained[4-8].

To sum up, this paper presents a hybrid artificial fish swarm optimization algorithm, the detection of global transactions and local affairs is obtained, the local affairs directly conflict detection is realized, and the deadlock is detected, the establishment of save points is taken, and the data recovery is obtained.

Principle of graphic database concurrency scheduling method and artificial fish swarm optimization algorithm

\section{A Algorithm principle}

In early twentieth Century, the experts in the relevant fields of database presents graphic database concurrency scheduling method based on artificial fish swarm algorithm, multi transaction 
execution problem can be optimized. But the advantages of this graphic database transaction scheduling method are relatively low, but also relatively simple, and the transaction accuracy requirements are selected. In order to shorten the convergence time scheduling, the graphics database transaction scheduling method is proposed based on artificial fish swarm algorithm, it has extensive application space. It can be used in many ways. However, this scheduling approach has certain defects. First, the correlation function of the dimension is relatively high, there must be a dimension, the dimensions should be reduced in processing. In addition, the function should be perfected. The conflict constraints can also cause rather big amount of computation, the detection spends a long time, finally influences the optimization effect.

Secondly, in the specified range, this method changes the database transaction scheduling approach without any rules, it needs for iterative processing, the accuracy of transaction scheduling processing is relatively low, and the speed is slow. Moreover, this algorithm is easy to cause the optimization efficiency is poor. Through the above exposition and analysis, it can be learned that the graphics database transaction scheduling method is proposed based on artificial fish swarm algorithm, it should also be improved. Therefore, a graphic database concurrency scheduling method is proposed based on hybrid artificial fish swarm algorithm, and the scheduling problem of massive graphics database is completed, the principle is expressed as follows:

Assumed the search space dimension of massive graphic databases is $\mathrm{n}$, the fish scale is $\mathrm{N}$. Each artificial fish can be represented as a n-dimensional vector $X_{i}=\left(\mathrm{x}_{i 1}, \mathrm{x}_{i 2}, \ldots, \mathrm{x}_{i n}\right)(i=1,2, \ldots, N)$, fitness function is $Y=f(\mathrm{x})$, it shows the real concentration of artificial fish in current state, $d_{i, j}=d\left(X_{i}, X_{j}\right)(i, j=1,2, \ldots, N)$ is the distance between artificial fish $X_{i}$ and artificial fish $X_{j}$, $\delta$ is the congestion factor, $0<\delta<1, N_{c r y}$ is the maximum number of artificial fish in each moving test. $R_{v i s}$ is the vision range of artificial fish in each moving test. The detailed steps are shown as follows:

(1) The initial population is generated by random way: the initial state of the fish is produced, assumed the a number of transactions required scheduling is $\mathrm{m}$, the number of scheduling objectives tasks is $\mathrm{N}$, the initial state is an artificial fish following random

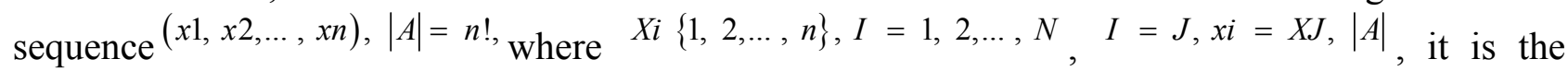
feasible solution set of elements in A.

(2) Fitness computation: fitness function converts the artificial fish state $X=\left(x_{1}, x_{2}, \ldots, x_{n}\right)$ into a scheduling scheme, the calculation results are obtained and return the transaction scheduling database weights. The fitting first heuristic algorithm is designed, achieve this transformation, in a "transaction scheduling priority scheduling database arrangement", the "priority" is selected, the specific steps of the rules are shown as follows:

(a) Keep the first effective transaction arrangement graphics database, the transaction selection is $b_{i}\left(S_{i}\right)$, the weight is taken as the first member in a group of fish, and set $U=\{S i\}$;

(b) In the affairs of the arrangement series, the remaining transaction $b_{j}\left(S_{j}\right)$ is selected, if $U \cap S_{j}=\Phi$, retains the $b_{j}\left(S_{j}\right)$, its "weight" is taken as the a member of the fish in the fish swarm, it will be added in the fish swarm, and set $U=U \cup\left\{S_{j}\right\}$. Otherwise, return to step (3).

(c) If $U=M$, exit; otherwise, take step (4); If $b_{j}\left(S_{j}\right)$ is a graphical database transaction arrangement in the final "weight", exit; otherwise, return to step (2).

(d) Foraging, assumed the current state of artificial fish is $X i$, select a state of $X_{j}$ randomly, where $X j=\{X \mid X A, D I J=R v i s, I=j\}$. If $Y_{i}^{<Y_{j}}$, then $X_{j}=X_{j}+C_{\text {step }}\left(X_{i}-X_{j}\right) / d_{i j}$, where, $C_{\text {step }}$ is the step size factor, $C_{\text {step }}=1$. otherwise, select the new state ${ }^{X j}$ randomly, When the number of 
randomly selection stats selected is larger than $N_{t r y}$, other actions (such as random movement) is carried out.

(3) Collision: Assumed the current state of artificial fish is $X_{i}$, and $M A X$

$X_{\max }=\quad X \in\left\{X \mid \mathrm{X} \in A, d\left(X, X_{i}\right) \leq R_{v i s}\right\} \quad, \quad \sum_{S \in X} \quad b(S) n_{f} \quad$ is the base of set $\left\{X \mid X \in A, d(X, X i) \leq R_{v i s}\right\}$. If $Y_{i}<Y_{\max }$, and $n f / N<\delta$, it shows there are enough food in XMAX, and it is not too crowded, then $X_{i}=X_{i}+C_{\text {step }}\left(X_{\max }-X_{i}\right) / d_{\max , i}$; Otherwise, the execution of foraging behavior is taken, this step can also rescue the defect constraints from conflict.

(4) Clustering: the current status of the artificial fish is $X_{i}, X_{c}$ is the center position of neighborhood of $X_{i}$, If $n f / N<\delta$, then $Y_{i}<Y_{C}$, then $X_{i}=X_{i}+C_{\text {step }}\left(X_{c}-X_{i}\right) / d_{c, i}$; otherwise, foraging behavior is taken.

\section{B Scheduling process description of graphics database concurrency}

The graphic database concurrency scheduling process is described as follows:

(1) Produce the initial transaction weights, and the constitution of a population is obtained.

(2) The fitting first heuristic algorithm is used to calculate the scheduling weights;

(3) For every artificial fish $i(i=1,2, \ldots, N)$, the following operations are taken.

For massive data calculation and judgment of volley scheduling, the mass data volley after state is superior to the original state, if it is positive, then turn step (4), or turn the step (2).

Cluster, judge whether the state is superior to the original state after execution, if it is null, then produce the initial transaction, the weights constitute a population are calculated.

Foraging, calculate the optimal weight iteration, calculate the scheduling weights, carry out the following operations, the optimal scheduling weights are updated in the current stage. Update the fish distance $i, j,(i, j=1,2, \ldots, N)$. If the largest number of iterative processing is reached, exit. Otherwise, turn (3).

The artificial fish $X=\left(x_{1}, x_{2}, \ldots, x_{n}\right), Y=\left(y_{1}, y_{2}, \ldots, y_{n}\right)$, then the distance between $\mathrm{X}$ and $\mathrm{Y}$ is shown as:

$$
\mathrm{d}(\mathrm{X}, \mathrm{Y})=\sum_{i=1}^{n} \operatorname{sign}\left(\left|\mathrm{x}_{i}-\mathrm{y}_{i}\right|\right)
$$

Where: $\quad(-1, x<0 \quad, \mathrm{k}-$ distance neighborhood can be expressed as: $N(X, k)=\{\mid d(, X)<k, \in D\}$. Dispatching affairs are $X_{1}, X_{2}, \ldots, X_{N}$, the scheduling center is expressed as follows:

$$
\operatorname{Center}\left(X_{1}, X_{2}, \ldots, X_{N}\right)=\underset{i=1,2, \ldots, \mathrm{N}}{\operatorname{Most}}\left(x_{1}^{i}, x_{2}^{i}, \ldots, x_{n}^{i}\right)
$$

Among them, the most operators take the most common or the most closely value. You can use new method to judge the scheduling performance, and the above method uses the fish foraging for improving the clustering performance, the mathematical model is proposed, it can solve constraint conflict defects, complete the transaction concurrency graphics database of optimal scheduling.

\section{Experiment results and analysis}

Although the traditional transaction scheduling algorithm can ensure global transaction commit order global serializability, but GTM know nothing at all about local affairs, it cannot detect the indirect conflict formation and local affairs, a new transaction scheduling is proposed, the hybrid artificial fish swarm algorithm fitting first heuristic algorithm and artificial fish swarm algorithm 
are combined, if there is no conflict, it can be selected according to the presence or absence of reasonable dispatching mode serialization, and use the save point to restore aborts the transaction.

In order to verify the validity of this algorithm, an experiment is taken. In the process of the experiment, the traditional algorithm and the improved algorithm are used in the graphics database transaction scheduling, the maximum concurrent number of transaction scheduling are calculated for comparison. The results are shown in table 1.

Table 1 Concurrency scale of different algorithms

\begin{tabular}{ccc}
\hline Number of transactions & $\begin{array}{c}\text { Concurrency of traditional } \\
\text { algorithm }\end{array}$ & $\begin{array}{c}\text { Concurrency value of new } \\
\text { algorithm }\end{array}$ \\
\hline 289 & 224 & 201 \\
426 & 267 & 394 \\
612 & 439 & 537 \\
\hline
\end{tabular}

The transaction amounts number is 787 , the traditional algorithm accommodate maximum concurrent number is 439 , the proposed method based on artificial fish swarm algorithm and priority heuristic algorithm has better performance in scheduling, the maximum concurrent quantity is 537, it is better than the traditional method. Therefore, this algorithm has certain advantages, the multi database transaction concurrent scheduling algorithm is proposed in this paper can improve the graphics database scheduling performance, and it can improve the efficiency of graphic database concurrency scheduling.

\section{Conclusions}

In this paper, the transaction scheduling problem of graphic database is researched. The reasonable arrangement of the database transaction commit is taken in order, and the fitting first heuristic algorithm and artificial fish swarm algorithm are combined. The graphic database transaction scheduling problem in the massive database is calculated. The foraging and clustering mathematical models are constructed in the artificial fish swarm, the constraint conflict drawbacks are solved. The optimal scheduling is obtained. The experimental results show that the scheduling problem of hybrid artificial fish swarm algorithm is solved, and the performance of massive database transaction scheduling is improved, the database transaction scheduling is quick and effective. The serializable processing of database transaction is ensured.

\section{Acknowledgments}

This work was financially supported by Science and Technology Research Project of Jilin Prov ince Education Bureau(201363), and Jilin Agricultural University Youth Foundation(201326)

\section{References}

[1] Guan X,Luh P B,Zhang L. Nonlinear approximation in Lagrangian relaxation-based fo hydrothermal scheduling[J].IEEE Transactions on Power Systems,2000,(02):772-778.

[2] Shawwash Z K,Siu T K,Russell S O D. The BC hydro short term hydro scheduling optimization model[J].IEEE Transactions on Power Systems,2000,(03):1125-1131.

[3] Abdelhalim M B,Habib S E-D. An integrated high-level hardware/software partitionin g methodology[J].Des Autom Embed Sys,2011,(01):19-50.

[4] Xing J T,Xiong Y P,Price W G. Passive-Active Vibration Isolation Systems to Prod uce Zero or Infinite Dynamic Modulus:Theoretical and Conceptual Design Strategies[J]. $\{\mathrm{H}\}$ Journal of Sound and Vibration,2005.615-636.

[5] INGLADA J,MURON V,PICHARD D. Analysis of artifacts in subpixel remote sensin $\mathrm{g}$ image registration[J].IEEE Transactions on Geoseience and Remote Sensing,2007,(01):254-264. 
[6] PLUIM J P W,MAINTZ J B A,VIERGEVER M A. Mutual information based registr ation of medical images:a survey[J].IEEE Transactions on Medical Imaging,2003,(08):9 86-1004.

[7]Rao Yutai,Yang fan. Network Intrusion Stir the Network Instability Control Methods of the Research[J]. BULLETIN OF SCIENCE AND TECHNOLOGY, 2014,30(1): 185-188.

[8]LI Feng, WU Chun- ming. Research on Prevention Fluctuation Control method of Network Intrusion Based on Energy Management[J]. Computer simulation, 2013,30(12): 45-48, 335. 